\title{
Pedunculopontine nucleus deep brain stimulation in Parkinson's disease
}

Tomasz Tykocki ${ }^{1}$, Tomasz Mandat², Paweł Nauman ${ }^{1}$

1Department of Neurosurgery, Institute of Psychiatry and Neurology, Warsaw, Poland
2Department of Neurosurgery, Maria Skłodowska-Curie Memorial Oncology Centre,
Warsaw, Poland

Submitted: 4 November 2010

Accepted: 14 April 2011

Arch Med Sci 2011; 7, 4: 555-564

DOI: 10.5114/aoms.2011.24119

Copyright (c) 2011 Termedia \& Banach
Corresponding author: Tomasz Tykocki MD Department of Neurosurgery Institute of Psychiatry and Neurology 9 Sobieskiego 02-957 Warszawa, Poland e-mail: ttomasz@mp.pl

\begin{abstract}
Postural instability and gait difficulty (PIGD) are commonly observed in advanced Parkinson's disease. The neuronal mechanism of PIGD is not fully understood. Dysfunction of the pedunculopontine nucleus (PPN) might be a possible cause of these symptoms. The autopsy studies of subjects with PIGD revealed a neurodegenerative process involving mainly PPN cholinergic neurons. The PPN participates in the locomotion processes by initiation, modulation and execution of stereotyped patterns of movement. The standard neurosurgical treatment of PD is subthalamic deep brain stimulation (STN DBS). Clinical results revealed low efficiency of STN DBS on PIGD. Preliminary results of simultaneous PPN and STN DBS are very promising. Only a few reports have been published until now; a significant improvement of PIGD was observed in both ON and OFF L-dopa states.
\end{abstract}

Key words: pedunculopontine nucleus, Parkinson's disease, deep brain stimulation.

\section{Introduction}

Parkinson's disease (PD) is a neurodegenerative disorder, which affects $1-2 \%$ of the population above 60 years old. It is estimated that around 5 million people worldwide [1] and 80 thousand in Poland suffer from PD [2]. Postural instability and gait difficulty (PIGD) [3] are the cardinal motor symptoms observed in $16 \%$ of PD patients [4]. Frequency of PIGD is higher in old-age onset of PD [5]. Pharmacological treatment, including l-dopa, frequently can cause significant side effects and becomes less effective with PD progression. Symptoms such as PIGD, freezing and dysautonomia are refractory to standard pharmacological treatment, which could be explained by progressive degeneration of the non-dopaminergic system in PD. Postural instability and gait difficulty are associated with the gait initiation phase $(\mathrm{Gl})$, which consists of two elements: the preparation period and a movement execution period [6]. Gait initiation uses stereotyped, coordinated patterns of limbs and trunk movements. Gait initiation difficulties are caused by two factors: (1) prolongation of gait preparation, execution interval and difficulty in recalling stereotyped, adequate motor programme sequences, and (2) impaired motor programme selection and coordination of the movements involved. In recent decades, the standard non-pharmacological treatment of PD has become deep brain stimulation (DBS) [7]. Subthalamic nucleus (STN) DBS 
is not as effective in PIGD as in other motor symptoms. It is speculated that PIGD may result from damage of non-dopaminergic systems, such as the mesencephalic locomotor region (MLR). Mesencephalic locomotor region is composed of the pedunculopontine nucleus (PPN) and the cuneiform nucleus, which contain both cholinergic and non-cholinergic neurons [8]. Pedunculopontine nucleus dysfunction may be responsible for PIGD in PD. It appears that PPN DBS is a promising new target for PIGD. Recent clinical results confirm the effectiveness of this therapeutic procedure [9].

The authors present a review of the literature on the application of PPN DBS for the treatment of PIGD in PD, including the anatomy and physiology of the PPN and clinical results.

\section{Anatomy of pedunculopontine nucleus}

The PPN is located in the dorsolateral part of the pontomesencephalic tegmentum, at the level of the trochlear nucleus. It is delimited by the superior cerebellar peduncles medially, by the medial lemniscus laterally and ventrally and by the cuneiform nucleus dorsally. The PPN is separated from the STN by the retrorubral field [10]. Histologically, the PPN is composed of two sub-regions: the pars compacta (CPPN) and pars dissipata (dPPN). The cPPN consists of a compact cluster of large neurons, located within the caudal half of the nucleus in its dorsolateral region. The dPPN is the main part of the PPN and arises in the superior cerebellar peduncle and tegmental tract, with uncertain boundaries [10]. There are around 10000 to 15000 cholinergic neurons within the PPN. Two subgroups of cholinergic, choline-acetyltransferase positive neurons have been identified. Most Ch5 neurons incorporate the PPN territory and Ch6 are

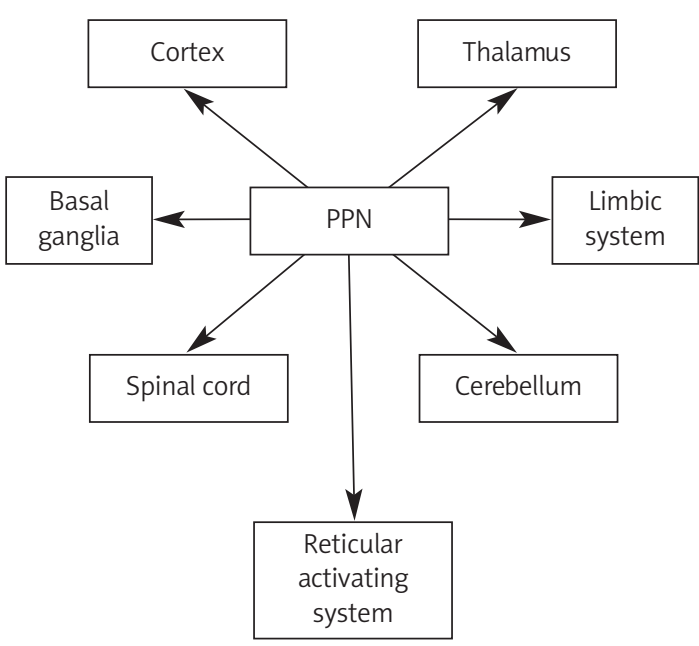

Figure 1. Connections of PPN with brain structures $P P N$ - pedunculopontine nucleus within the area corresponding to the lateral dorsal tegmental nucleus [11]. Physiologically, three types of cholinergic cells have been identified: 1) low threshold spikes cells; 2) cells with a transient outward current; 3 ) and cells with both characteristics [12]. There is a wide spectrum of receptor heterogeneity in the PPN cholinergic system. Fifty percent of the neurons express $\alpha 2 \mathrm{a}$ and $30 \% \alpha \mathrm{a} 1$ receptors. Some of the cholinergic cells co-release substance $P$, enkephalin, calbindin, calretinin and parvalbumin. The non-cholinergic neurons belong to the glutaminergic and GABAergic system [13].

Anatomical and electrophysiological studies have revealed numerous putative connections with brain structures including the cortex, limbic structures, basal ganglia, thalamus, brainstem, cerebellum and spinal cord (Figure 1). The PPN is closely functionally related with the reticular activating system, by its cholinergic and glutaminergic connections with intralaminar thalamic nuclei resulting in global activation of the brain. Chemical or electrical stimulation of PPN is followed by prolonged depolarization of thalamocortical cells and gamma oscillations in EEG. These electrophysiological changes are similar to those observed in wakefulness and REM sleep [14]. Pedunculopontine nucleus receives descending projections from the ipsilateral prefrontal and motor cortex. The corticotegmental tract, intermingled with the pyramidal tract, descends to the level of the substantia nigra, where they leave the cerebral peduncle and target the PPN. Cortico-PPN connections are organized somatotopically from medial to lateral (orofacial, forelimb, hindlimb) [15]. Cortical afferents are mainly glutaminergic. Limbic structures (amygdale, hippocampus and cingulated gyrus) send indirect efferent fibres to the PPN via the nucleus accumbens, ventral tegmentum area and ventral pallidum [16].

The most important and complex connections of the PPN are those formed by reciprocal pathways with basal ganglia, especially with the internal globus pallidus (GPi) and substantia nigra (SN). Globus pallidus and SN send the major afferents to the PPN by GABAergic transmission. Pallidal fibres descend along the pallidotegmental tract, originating from the medial segment of the GPi and terminating in the non-cholinergic cells of the dPPN [17]. These inhibitory pathways efferent mainly to the ipsilateral PPN, and only $10-20 \%$ pass to the contralateral PPN. Over $80 \%$ of pallidal neurons send collaterals to the ventrolateral nucleus of the thalamus. Animal studies have revealed pedunculopallidal fibres, passing along both the ansa lenticularis and lenticular fascilulus [18]. Both SNr (substantia nigra, pars reticulata) and SNc (substantia nigra, pars compacta) send projections to the PPN (Figure 2). These fibres divide into two 
branches, one nigro-tectal, terminating in the deeper layer of the superior colliculus, and the second, the smaller part in the PPN $[19,20]$. The PPN sends direct efferent, glutaminergic and cholinergic connections to the SNc and indirect connections via the STN to the SNr. The STN innervates the PPN by direct connections originating in the lateral STN and indirect connections by collaterals of STN-GP and STN-SN pathways $[20,21]$. Numerous thalamic projections, especially to the centrolateral, ventrolateral and reticular nucleus, pass through the PPN. The PPN-thalamic pathways control thalamic activity and pass to the striatum, as part of the subcortical-thalamus-basal ganglia-subcortical loop [22, 23]. Other identified PPN-striatum connections proceed through midbrain dopaminergic neurons. Projections forming the anterior PPN region run to the SNc and those from the posterior region terminate in the ventral tegmental area. The collaterals of PPN-thalamic pathways are the main source of dopaminergic innervations for midbrain neurons [24, 25]. There are two main brainstem structures connected with the PPN: brainstem reticular nuclei (pontine reticular nuclei oralis and caudal, ventromedial portion of gigantocellular nucleus) and periaqueductal grey matter [26]. The PPN projections to the cerebellar fastigial nucleus, red nucleus and spinal cord play an important role in the genesis of axial symptoms in PD [27, 28].

A study with diffusion tensor imaging (DTI) [28] in 8 volunteers found multiple neural connections previously identified in animals. Pathways between the PPN and primary motor cortex had features of heterogeneity among the participants. Connections with the upper extremity were present in $87.5 \%$ of patients, the lower extremity in $62.5 \%$, the trunk in $56.25 \%$, and the orofacial region in only $12.5 \%$ of patients. All these connections passed via the posterior limb of the internal capsule. In $75 \%$ of volunteers, pathways to the cerebellum via the superior cerebellar peduncle were shown, comparably with monkey studies. In all cases connections with the spinal cord were noted. Comparison of human and monkey (Macaca mulatta) PPN connectivity determined using probabilistic diffusion tractography (PDT) and histological tracing techniques revealed certain anatomical dissimilarities [29]. Human PPN connections with SN were displayed in less than half of all subjects; the same tracts are more prominent in monkeys. Other differences could be noted in the reciprocal functional organization and representation of primary motor cortex and PPN. This anatomical discrepancy may reflect the human bipedal and standing posture and quadrupedal gait in primates.

Neuroimaging of gait with $\mathrm{fMRI}$ showed an increased BOLD signal in MLR with the overall

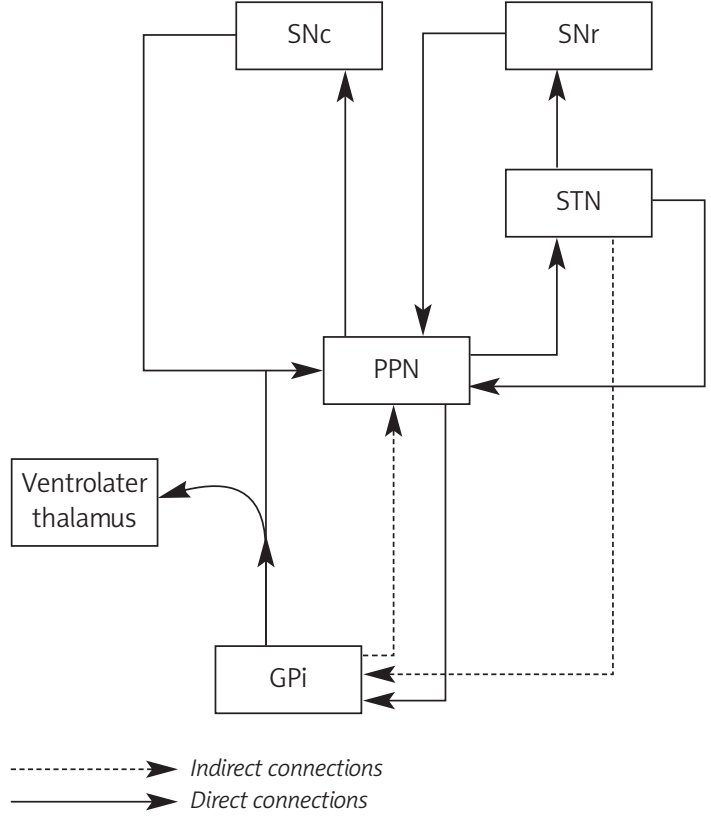

Figure 2. Connection of PPN with basal ganglia

$P P N$ - pedunculopontine nucleus, SNr-substantia nigra, pars reticulata, $S N c$ - substantia nigra, pars compacta, GPi - internal globus pallidus

reduction of BOLD activity in basal ganglia of $P D$ patients. Opposite effects were observed in the control group [30]. Single photon emission computed tomography with [99mTc] hexamethylpropyleneamine oxime during gait on a treadmill revealed increased regional cerebral blood flow ( $\mathrm{rCBF}$ ) in MLR. These findings were considered as characteristic only for quadrupedal animals [31].

The PET study with 15-O-labelled CO demonstrated diminished regional cerebral blood flow of the supplementary motor area associated with hypokinesia in PD. These findings were observed only during voluntary movements; no rCBF deviation appeared during triggered movements. Hypothetically, distinct motion regulative systems could be mediated by the connections from the globus pallidus internus via the pedunculopontine nucleus to the cerebellum [32].

\section{Functional anatomy of pedunculopontine nucleus}

The PPN is part of the MLR, which is functionally defined as the area of the brainstem responsible for locomotion. The outputs of the MLR are bilaterally distributed to the reticulospinal cells in the ventromedial medulla, with an ipsilateral predominance. The MLR descending projections enable coordination of limbs and postural movements. The physiological analysis of PPN function is mainly based on animal studies. The human analysis with fMRI confirmed increased 
activity of the left PPN in right-handed healthy subjects during imaging of gait motion. However, this study presents only the indirect evidence of locomotor involvement of PPN, because obtaining fMRI during gait motion is technically impossible [33]. Electrical stimulation at $50-60 \mathrm{~Hz}$ near the PPN in chronic pain patients revealed increased tone in the contralateral limb muscles [34]. Three groups of functional PPN cells were distinguished: 1) 'on' cells - with tonic firing activity on locomotion and stopped entirely with the cessation of motion: 2) 'off' cells - displaying a tonic firing pattern with frequency increase before motion cessation; 3) 'bursters' - displaying a bursting pattern of firing on locomotion. The on/off cells modulate the duration of locomotion, while the bursters control the intensity and initiation of gait [35]. Unilateral PPN kainic acid or radiofrequency lesions in the animal studies resulted in contralateral hemiparkinsonism [36].

The PPN cholinergic neurons are part of the motivation and reward circuit and are combined with the limbic and brainstem motivational system. The PPN receives inputs from limbic and striatum, acting as a selective and coordinative centre for the affective impulses, preparing a programmed motor reaction, appropriate to the emotional status [37]. The PPN is afferently connected with the dorsolateral ventral pallidum (dVP) and sends two projections: 1) to the spinal cord through the brainstem motor nuclei; 2) to the ventral tegmentum area, which mediate dopaminergically to the nucleus accumbens and dVP, which close the loop [38].

Post-stimulation (PPN DBS) DTI results showed normalization of cortical and brainstem connectivity in one analysed PIGD patient. Previously absent cerebellar connectivity was now restored. The abnormal, anterior pontine connectivity was reduced and reorganized, so that the dominant connectivity was with the pre-frontal cortical areas, instead of the primary motor cortex [39]. Discrepancy of PPN connection in different subtypes of PD patients was found. Imaging with DTI FA detected absence of PPN connectivity to any part of the cerebellum in PIGD patients, whereas the non-PIGD PD group displayed significant connectivity with the cerebellum, similar to, although not completely overlapping with healthy normal controls. The PIGD patients showed anterior pontine connectivity at the level of the mid-section of the fourth ventricle, which was absent in non-PIGD PD patients and controls. These results support the central role of the cerebellum in the pathomechanism of PIGD. Increased activity of the anterior pons may reflect the compensatory, enhanced corticopontocerebellar projections in PIGD patients [40]. 18-fluorodeoxyglucose (FDG)-positron emission tomography (PET) of $6 \mathrm{PD}$ patients during
PPT DBS showed significantly increased FDG uptake in prefrontal and frontal bilateral cortical areas, including superior and inferior frontal gyri, dorsolateral prefrontal and orbitofrontal cortex, anterior cingulated, supramarginal gyrus and inferior parietal lobule. Moreover, PPN DBS induced increased FDG consumption in the left ventral striatum, right insula and right superior temporal gyrus. These findings stress the involvement of the PPN not only in movement coordination, but some participation in cognitive, emotional and executive functions [41]. A [15O] $\mathrm{H}_{2} \mathrm{O}$ PET study showed that unilateral PPN stimulation induced a significant regional cerebral blood flow increase in subcortical regions such as the cerebellum, thalamus bilaterally and MLR. Additionally, PPN DBS during motor limb movement resulted in increased rCBF in the medial sensorimotor cortex extending into the caudal supplementary motor area. Presumably, PPN modulation may induce functional changes in neural networks associated with the control of lower limb movements [42].

The rapid eye movement (REM) sleep state is characterized by a desynchronized electroencephalogram. Physiologically, the REM mechanism is a complex process, involving various neurotransmitters and coordination of the appropriate brain structure. The mesopontine reticular activating system (RAS), which modulates the sleep-wake cycle, is composed of PPN, locus coeruleus and raphe nucleus. All these three structures act in functionally reciprocal connections. During the waking state all these structures are active. The PPN cholinergic neurons decrease firing during slow-wave sleep; they become active during wake and REM state, that is in fast cortical rhythms [43].

The PPN is also a part of primary acoustic startle reactions. A single acoustic stimulus activates the ventral cochlear nucleus and PPN, which send cholinergic inhibitory signals to the nucleus reticularis pontis caudalis. The PPN modulates and regulates the sensorimotor gating information from the forebrain [44].

\section{Neuropathology of pedunculopontine nucleus}

The PIGD may reflect the loss of neurons and dysfunction of the PPN in PD patients. However, there are no data explaining whether the neurodegenerative processes of SN and PPN are of the same kind. Some authors report that 40-50\% of cholinergic neurons degenerate in PD. Lewy bodies were observed in remaining PPN cells, indicating primary neurodegeneration [45]. The loss of catecholaminergic cells has also been determined. The decrease of cholinergic cells may result in overactivity of intact glutaminergic neurons [46], which innervate the dopaminergic SNc region. This effect accelerates or even causes the nigral 
degeneration. Pallidal-nigral projections are mainly inhibitory and in consequence lead to expanded PPN inhibition [47]. Therefore, there are two possible mechanisms of PPN insufficiency, neurodegeneration and functional inhibition. There is no correlation between PD duration and extent of PPN neuronal loss; also, intact PPN structures in PD brains were found [45]. That is in contradiction to animal models, which revealed that MPTP treatment induced 30\% more expanded loss of cholinergic neurons in aged subjects, in comparison with young ones [33].

Karachi et al. [33] analysed post mortem brain sections of three groups: 1) PD patients without balance deficit and falls (non-faller, nfPD); 2) PD patients with balance deficits and falls (faller, fPD); 3) healthy controls. The number of cholinergic cells in fPD patients was significantly lower than in nfPD and controls. The loss of dopaminergic neurons was also more extensive in fPD than in nfPD. There was no difference in the number of cuneiform nucleus cells in both PD groups, which indicates that PPN impairment rather than the extent of degeneration is the cause of PIGD. Bilateral lesions of PPN in animal models resulted in significant changes in gait and posture at 7-8 weeks follow-up. No changes of global motor activity was observed and the symptoms did not improve after application of apomorphine [33]. Simultaneous PPN local field potentials and cortical EEG showed a correlation between motor cortex activity and PPN. Dopaminergic medications modulate PPN synchronization with voluntary movements and the $\mathrm{ON}$ state promotes cortical-PPN interactions [48]. A PET study in nfPD and fPD patients showed significantly lower cholinergic activity in the fPD group. Cholinergic dysfunction seems to be crucial for PIGD and is a possible target for pharmacological intervention. Administration of scopolamine, an antimuscarinic agent, has been reported to worsen gait in progressive supranuclear palsy (PSP) and elderly patients $[49,50]$. There is preliminary evidence that varenicline selective $\alpha 4 \beta 2$, a nicotinic receptor agonist, may have a beneficial effect on gait; promising results have been reported in patients with ataxia [51]. Studies have also shown a positive association between PIGD in PD and increased risk of dementia [52, 53]. However, there are no studies investigating and answering whether the cholinergic pathology of PIGD and PD dementia are of the same kind.

\section{Pedunculopontine nucleus deep brain stimulation}

Ablative techniques and deep brain stimulation are recommended for surgical treatment of PD. Neuromodulation with DBS includes such targets as the STN, nucleus ventralis intermedius thalami
(Vim) and globus pallidus pars interna (GPi). It is believed that STN DBS produces the widest effect on motor symptoms in PD. Nevertheless, the benefits in PIGD after STN DBS are no so promoting and tend to decline with the natural course of the disease. Previous animal studies and pioneer clinical trials of low frequency PPN DBS showed significant amelioration of gait and postural deficits in PD. Jenkinson et al. [54] reported the effects of unilateral PPN stimulation in macaques before and after inducing parkinsonism with MPTP (1-methyl-4phenyl-1,2,3,6-tetrahydropyridine). Stimulation at frequency $5 \mathrm{~Hz}$ showed increased motor activity in MPTP monkeys comparable to that observed after levodopa treatment. Similar results were noted in normal macaques. Another animal study with the 6-hydroxydopamine (6-OHDA) rat model investigated the reciprocal interactions in basal ganglia circuits. In 6-OHDA rats the STN and SNr are hyperactive and after unilateral ibotenic acid lesioning of PPN the activity of both the STN and $\mathrm{SNr}$ returned to the baseline [55]. However, Rauch et al. [56] in a detailed analysis of PPN stimulation in a 6-OHDA rat model reported a non-uniform response to high and low frequencies according to different motor functions: locomotor activity, postural instability and limb use. These complex responses to PPN stimulation require further investigations. A trial with microinjection of bicuculline, a GABA A antagonist, into the PPN of parkinsonian monkeys clearly proved alleviation of akinesia [57].

The first clinical reports of benefits with PPN DBS come from 2005, when Mazzone et al. [58] and Plaha et al. [59] published their results (Table I). Bilateral PPN DBS alone, without previous STN DBS or simultaneous implantation or stimulation of STN, revealed improvement in the total Unified Parkinson's Disease Rating Scale (UPDRS) - 53\% and UPDRS part III (motor subscore) - 57\% and levodopa reduction of $32.5 \%$. The two selected patients had dominant symptoms of PIGD and freezing. Postoperative follow-up was performed at day 42 for the first patient and day 16 for the second one. The best clinical response was observed at frequencies between $20 \mathrm{~Hz}$ and $25 \mathrm{~Hz}$ [59]. Electrophysiological recordings directly from the PPN during synchronous stimulation of STN produced only slight changes in PPN firing activity, while the same stimulation provoked increased $\mathrm{SNr}$ activity. These findings demonstrate that the STNPPN pathway in humans is not so extensive as is observed in rodents and cats [58]. Stefani et al. [60] proposed simultaneous bilateral implantation of DBS electrodes in STN and PPN. PD patients with PIGD dominancy were included with UPDRS (Unified Parkinson's Disease Rating Scale) part III score over 70. In OFF medication state the following 


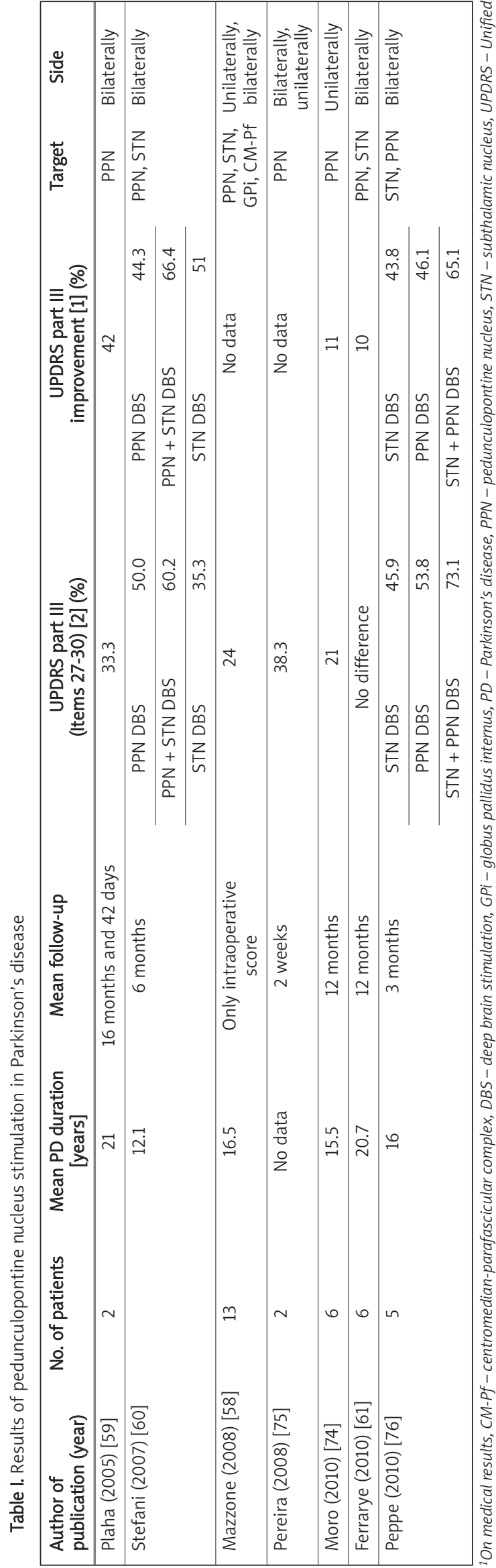

reduction of UPDRS III was reported: $33 \%$ PPN DBS, $54 \%$ STN DBS, 56\% STN plus PPN DBS. Including only the axial signs (points 27-30 of UPDRS III), PPN DBS was more effective than STN DBS alone but less than STN plus PPN DBS. No significant differences among the three DBS conditions were detected. The combination of L-dopa treatment with DBS produced a significant reduction of the UPDRS score (PPN DBS 72.4\%, STN DBS 75.8\%, PPN and STN DBS $83.5 \%$ ) in comparison with any OFF medication stimulation or with L-dopa alone (50.2\%). The PIGD improvement after PPN stimulation in the ON medication state seems to be unusual, considering that axial symptoms are generally ascribed to 'non-dopaminergic' degeneration. Ferraye et al. [61] presented results of PPN DBS for PIGD with a double-blind, cross-over stimulation study. Six patients were recruited with refractory PIGD and previous STN DBS. Results were ambiguous: while some patients significantly improved after PPN DBS, others obtained moderate benefits and one even worsened within one year after the operation. Regarding the whole group, freezing improved ON stimulation in comparison with OFF stimulation, in both OFF and ON medication states. Outcomes in the Beck Depression Inventory and mobility subscale of PDQ-39 (Parkinson's disease questionnaire) ameliorated after surgery, but did not achieve statistical significance. The PIGD did not respond to the stimulation. Therefore, these observations could be provoked by combined stimulation of cholinergic and glutaminergic PPNC and PPNd cells, and indirectly facilitating the dopaminergic striatal system. Intraoperative recordings showed that PPN DBS $(25 \mathrm{~Hz}, 60 \mu \mathrm{s})$ affects the STN firing rate. Two conflicting STN neuronal responses were observed after PPN DBS: decreased firing in bursting neurons and an excitatory effect in large tonic neurons. Silence in burst neurons is in accord with a PPN therapeutic effect, but increased tonic mode is not desired [62]. Stimulation at frequency of $180 \mathrm{~Hz}$ resulted in motor deterioration. PPN DBS modulates descending basal ganglia-brainstem pathways. The PD patients present an increased Hoffman reflex (HR) threshold; this abnormality was significantly reduced by DBS. In particular, it was found that PPN-DBS alone, or plus STN-DBS, induced complete normalization of the HR threshold [63]. The PPNDBS may act on spinal cord excitability by improving s the reticulospinal pathway transmission; thus the effectiveness of the stimulation might be due not only to the modulation of the basal ganglia circuits, but also excitation of the pontomedullary reticular formation and reticulospinal nuclei improving descending spinal pathways. The PET imaging in PD patients with unilateral PPN DBS revealed increased regional blood flow in the thalamus, 
cerebellum, midbrain region and cortical areas involving the medial sensorimotor cortex extending into the caudal supplementary motor area [64]. The PPN-DBS significantly improved executive functions and working memory and reduced sleep fragmentation and comfort. This effect is probably due to activation of cholinergic muscarin receptor transmission from the PPN to the thalamus.

\section{Prospects for the future}

Deep brain stimulation was introduced in the 1990s. Since then, DBS has become the standard therapy in the treatment of movement disorders. Neuromodulatory procedures for the treatment of PD appeared to be clinically effective, as they decrease the severity of dominant symptoms. Nevertheless there is currently no evidence that DBS inhibits the progression of the neurodegenerative process. In the course of PD, apart from the cardinal motor signs, dysautonomia and psychiatric symptoms are also distinguished. In the treatment of these so-called non-motor symptoms, DBS seems to be ineffective. Besides, there is also a group of patients who do not respond to the neuromodulatory treatment for motor disturbances, in particular those with severe axial symptoms such as PIGD. Thus, investigations have been started to find new potential anatomical and physiological targets to increase the effectiveness of DBS in PD. One of the promising targets is the PPN, and the simultaneous stimulation of the PPN and STN proved effective in the treatment of PIGD. Further exploration of an appropriate strategy in the neuromodulatory therapy, considering the selection of single or combined targets depending on the dominance of symptoms in PD, may potentiate the effectiveness of DBS. Great expectations are associated with other therapeutic approaches, such as stem cells [65] or gene therapy [66] in the treatment of PD. Transplantation of embryonic dopamine neurons for severe PD seemed to be clinically very effective. However, promising initial motor results were related to serious graft-induced dyskinesias [67]. Further investigation elucidated that the possible cause of these side effects is the impact of grafted serotonin neurons, as the serotonin neurons lack the autoregulatory feedback control typical for dopaminergic cells. In PD the physiological dopamine transmission in the striatum is possibly replaced by serotonin neurons, resulting in uncontrolled dopamine release. Animal studies demonstrate that graft-induced dyskinesias are evoked by high content of serotonin neurons in the graft. However, at least $10-20 \%$ of non-degenerative dopaminergic striatal neurons are sufficient to protect against the dyskinesias generated by new serotoninergic innervations. Therefore, human studies with respect to the aforementioned results are needed [68].
Gene therapy is a new concept for alleviation of symptoms and alteration of the progression of the disease. A double-blind, randomized study with glial cell line-derived neurotrophic factor (GDNF) via an adeno-associated type-2 vector did not confirm a significant advantage for gene therapy in comparison with other methods in the early followup [69]. A previous animal and human open-label trial [70] showed significant improvement in motor scales with no serious side effects. Considering the multiple degenerative brain process in PD, multitargeted GDNF delivery could be the solution for better clinical outcomes. However, the authors emphasize that in the longer observation gene therapy may be more effective.

High-resolution brain imaging using 7.0 T magnets could dramatically improve the accuracy of the target visualization and provide detailed information about the topography of the desired anatomical area [71]. Ultra high-field MRI scanning together with intraoperative real-time neurochemical monitoring and neurophysiological recordings may optimize not only the anatomical targeting but also greatly improve the functional navigation. The development of the Wireless Instantaneous Neurotransmitter Concentration System (WINCS) [72] for neurochemical monitoring, which can interface with carbon-fibre microelectrodes or enzyme-based microsensors to measure extracellular concentrations of neurotransmitters and neuromodulators. Fast-scan cyclic voltammetry (FSCV) generates a voltammogram, which is sent to a remotely located station where the data are analysed and chemical identification takes place. Real-time neurochemical monitoring of the targeted regions may provide a new strategy for investigation of DBS mechanisms and chemically navigated placement of the stimulating electrode. Another innovation, closed-loop responsiveness stimulation, is an alternative for intelligent, symptom-related stimulation [73]. This pattern of DBS has already been introduced in epilepsy treatment. The system is based on the feedbackguided adjustment of stimulation calibrated with neurophysiological and neurochemical monitoring from the targeted area, for example PPN. Upon the detection of abnormalities on monitoring, responsive electrical stimulation is delivered.

Future therapeutic options could be based on an individual strategy taking into account combined or single therapy considering pharmacotherapy, neuromodulation or gene therapy.

\section{Rationale for the application of pedunculopontine deep brain stimulation}

The PPN was proposed as a new stereotactic target for the neurosurgical treatment of PD. There have been published only a few reports evaluating 
clinical results of PPN DBS in approximately 30 patients so far. The main reason supporting this therapeutic approach is the significant improvement of axial symptoms in PD, in particular freezing of gait, frequency of gait and postural instability. Hitherto prevailing targets such as the STN or GPi used for DBS tellingly ameliorated the cardinal motor parkinsonian symptoms, but were inefficient in the treatment of axial problems. However, application of PPN DBS alone proved to be less effective than STN DBS or GPi DBS for nonaxial symptoms, but simultaneous stimulation of PPN and STN brought the best results for the treatment of PIGD. Since the disability associated with PIGD significantly affects the quality of life, PPN DBS offers a new prospect to improve comfort of PD patients. Also noteworthy is the improvement of cognitive, emotional and executive functions after PPN DBS. Additionally, a decrease in awakenings and a significant increase in REM sleep were observed after stimulation. Simultaneous bilateral PPN and STN DBS entails the need for implantation of eight electrodes, which increase the risk of surgical complications. Therefore, only selected PD candidates with PIGD diagnosed by a neurologist with experience in movement disorders should be qualified for PPN DBS. To date, only outcomes of pilot studies have been reported, and larger, double-bind design series are necessary to confirm these first promising results.

\section{Conclusions}

The PIGD belong to the dopamine-resistant symptoms. Although the effectiveness of STN DBS for cardinal motor symptoms is well documented, PIGD treatment is still unsolved. The PPN was discovered as a central structure responsible for coordination of locomotor functions. The PPN controls basal ganglia circuits and descending brainstem pathways. According to the animal models and the complex mechanism of action, the PPN was identified as a target for DBS in PIGD treatment. The performance of combined STN and PPN DBS treatment has shown its efficacy. The twotarget stimulation seemed more effective than the stimulation of each target alone. The unsatisfactory results of PPN DBS as a single target for PD symptoms suggest that it can be explored only as an additional target. The PPN DBS increased the efficiency of both STN DBS and pharmacological therapy used alone. The slight decline of PPN DBS efficacy in PIGD over months raises the possibility of adaptive changes, but longer follow-up is needed to confirm this observation. The fundamental issue is appropriate selection of patients for STN plus PPN DBS. PPN DBS is dedicated to PD patients with PIGD.

\section{Acknowledgments}

All authors have no conflict of interest to declare.

\section{References}

1. de Lau LM, Breteler MM. Epidemiology of Parkinson's disease. Lancet Neurol 2006; 5: 525-35.

2. Friedman A. Epidemiologia. In: Choroba Parkinsona, mechanizmy, rozpoznawanie, leczenie [Polish]. Friedman A (ed.). Wydawnictwo Czelej, Lublin 2005; 1-5.

3. Jankovic J, McDermott M, Carter J. Variable expression of Parkinson's disease: a base-line analysis of the DATATOP cohort. The Parkinson Study Group. Neurology 1990; 40: 1529-34.

4. Rajput $A H$, Pahwa $R$, Pahwa $P$, et al. Prognostic significance of the onset mode in parkinsonism. Neurology 1993; 43: 829-30.

5. Gibb WRG, Lees AJ. A comparison of clinical and pathological features of young- and old-onset Parkinson's disease. Neurology 1988; 38: 1402-6.

6. Brenière Y, Do MC. Control of gait initiation. J Mot Behav 1991; 23: 235-40.

7. Rosin R, Topka H. Dichgans gait initiation in Parkinson's disease. J Mov Disord 1997; 12: 682-90.

8. Garcia-Rill E. The pedunculopontine nucleus. Prog Neurobiol 1991; 36: 363-89.

9. Hamani C, Stone S, Laxton A. The pedunculopontine nucleus and movement disorders: anatomy and the role for deep brain stimulation. Parkinsonism Relat Disord 2007; 13 Suppl 3: S276-80.

10. Olszewski J, Baxter D. Cytoarchitecture of the human brain stem. J. B. Lippincott Co., Philadelphia 1954.

11. Mesulam MM, Mufson EJ, Wainer BH, et al. Central cholinergic pathways in the rat: an overview based on an alternative nomenclature (Ch1-Ch6). Neuroscience 1983; 10: 1185-201.

12. Saitoh K, Hattori S, Song WJ. Nigral GABAergic inhibition upon cholinergic neurons in the rat pedunculopontine tegmental nucleus. Eur J Neurosci 2003; 18: 879-86.

13. Hou YP, Manns ID, Jones BE. Immunostaining of cholinergic pontomesencephalic neurons for alpha 1 versus alpha 2 adrenergic receptors suggests different sleep-wake state activities and roles. Neuroscience 2002; 114: 517-21.

14. Steriade M, Dossi RC, Nuñez A. Network modulation of a slow intrinsic oscillation of cat thalamocortical neurons implicated in sleep delta waves: cortically induced synchronization and brainstem cholinergic suppression. J Neurosci 1991; 11: 3200-17.

15. Monakow KH, Akert K, Künzle H. Projections of precentral and premotor cortex to the red nucleus and other midbrain areas in Macaca fascicularis. Exp Brain Res 1979; 34: 91-105.

16. Swanson LW. The projections of the ventral tegmental area and adjacent regions: a combined fluorescent retrograde tracer and immunofluorescence study in the rat. Brain Res Bull 1982; 9: 321-53.

17. Lavoie B, Parent A. Pedunculopontine nucleus in the squirrel monkey: projections to the basal ganglia as revealed by anterograde tract-tracing methods. J Comp Neurol 1994; 344: 210-31.

18. Moriizumi T, Hattori T. Separate neuronal populations of the rat globus pallidus projecting to the subthalamic nucleus, auditory cortex and pedunculopontine tegmental area. Neuroscience 1992; 46: 701-10.

19. Steininger TL, Rye DB, Wainer BH. Afferent projections to the cholinergic pedunculopontine tegmental nucleus and 
adjacent midbrain extrapyramidal area in the albino rat. I. Retrograde tracing studies. J Comp Neurol 1992; 321: 515-43.

20. Edley SM, Graybiel AM. The afferent and efferent connections of the feline nucleus tegmenti pedunculopontinus, pars compacta. AM J Comp Neurol 1983; 217: 187-215.

21. Hammond C, Rouzaire-Dubois B, Féger J, et al. Anatomical and electrophysiological studies on the reciprocal projections between the subthalamic nucleus and nucleus tegmenti pedunculopontinus in the rat. Neuroscience 1983; 9: 41-52.

22. Ainge JA, Jenkins TA, Winn P. Induction of c-fos in specific thalamic nuclei following stimulation of the pedunculopontine tegmental nucleus. Eur J Neurosci 2004; 20 : 1827-37.

23. McHaffie JG, Stanford TR, Stein BE, et al. Subcortical loops through the basal ganglia. Trends Neurosci 2005; 28: 401-7.

24. Mena-Segovia J, Winn P, Bolam JP. Cholinergic modulation of midbrain dopaminergic systems. Brain Res Rev 2008 58: 265-71

25. Oakman SA, Faris PL, Kerr PE. Distribution of pontomesencephalic cholinergic neurons projecting to substantia nigra differs significantly from those projecting to ventral tegmental area. J Neurosci 1995; 15: 5859-69.

26. Skinner RD, Kinjo N, Ishikawa Y, et al. Locomotor projections from the pedunculopontine nucleus to the medioventral medulla. Neuroreport 1990; 1: 207-10.

27. Ruggiero DA, Anwar M, Golanov EV, et al. The pedunculopontine tegmental nucleus issues collaterals to the fastigial nucleus and rostral ventrolateral reticular nucleus in the rat. Brain Res 1997; 760: 272-6.

28. Muthusamy KA, Aravamuthan BR, Kringelbach ML, et al. Connectivity of the human pedunculopontine nucleus region and diffusion tensor imaging in surgical targeting. Neurosurgery 2007; 107: 814-20.

29. Aravamuthan BR, Stein JF, Aziz TZ. The anatomy and localization of the pedunculopontine nucleus determined using probabilistic diffusion tractography. Br J Neurosurg 2008; 22 Suppl 1: S25-32.

30. Snijders AH, Leunissen I, Bakker M, et al. Gait-related cerebral alterations in patients with Parkinson's disease with freezing of gait. Brain 2011; 134: 59-72.

31. Hanakawa T, Katsumi Y, Fukuyama H, et al. Mechanisms underlying gait disturbance in Parkinson's disease: a single photon emission computed tomography study. Brain 1999; 122: $1271-82$

32. Jahanshahi M, Jenkins IH, Brown RG, et al. Self-initiated versus externally triggered movements. I. An investigation using measurement of regional cerebral blood flow with PET and movement-related potentials in normal and Parkison's disease subjects. Brain 1995; 118: 913-33.

33. Karachi C, Grabli D, Bernard FA, et al. Cholinergic mesencephalic neurons are involved in gait and postura disorders in Parkinson disease. J Clin Invest 2010; 120 2745-54.

34. Young RF, Tronnier V, Rinaldi PC. Chronic stimulation of the Kölliker-Fuse nucleus region for relief of intractable pain in humans. J Neurosurg 1992; 76: 979-85.

35. Garcia-Rill E, Skinner RD. Modulation of rhythmic function in the posterior midbrain. Neuroscience 1988; 27: 639-54.

36. Aziz TZ, Davies L, Stein J, et al. The role of descending basal ganglia connections to the brain stem in parkinsonian akinesia. Br J Neurosurg 1998; 12: 245-9.

37. Inglis WL, Winn P. The pedunculopontine tegmental nucleus: where the striatum meets the reticular formation. Prog Neurobiol 1995; 47: 1-29.
38. Graybiel AM, Aosaki T, Flaherty AW, et al. The basal ganglia and adaptive motor control. Science 1994; 265: 1826-31.

39. Schweder PM, Joint C, Hansen PC, Green AL, Quaghebeur G, Aziz TZ. Post-stimulation PPN connectivity chronic pedunculopontine nucleus stimulation restores functional connectivity. Neuroreport 2010; 21: 1065-8.

40. Schweder PM, Hansen PC, Green AL, Quaghebeur G, Stein J, Aziz TZ. FOG patients showed connectivity of the pedunculopontine nucleus in parkinsonian freezing of gait. Neuroreport 2010; 21: 914-6.

41. Stefani A, Pierantozzi M, Ceravolo R, Brusa L, Galati S, Stanzione P. Deep brain stimulation of pedunculopontine tegmental nucleus (PPTg) promotes cognitive and metabolic changes: a target-specific effect or response to a low-frequency pattern of stimulation? Clin EEG Neurosci 2010; 41: 82-6.

42. Ballanger B, Lozano AM, Moro E, et al. Cerebral blood flow changes induced by pedunculopontine nucleus stimulation in patients with advanced Parkinson's disease: a [(15)O] H2O PET study. Hum Brain Mapp 2009; 30: 3901-9.

43. Heister DS, Hayar A, Garcia-Rill E. Cholinergic modulation of GABAergic and glutamatergic transmission in the dorsal subcoeruleus: mechanisms for REM sleep control. Sleep 2009; 32: 1135-47.

44. Koch $M$, Kungel $M$, Herbert $H$. Cholinergic neurons in the pedunculopontine tegmental nucleus are involved in the mediation of prepulse inhibition of the acoustic startle response in the rat. Exp Brain Res 1993; 97: 71-82.

45. Hirsch EC, Graybiel AM, Duyckaerts C, Javoy-Agid F. Neuronal loss in the pedunculopontine tegmental nucleus in Parkinson disease and in progressive supranuclear palsy. Proc Natl Acad Sci U S A 1987; 84: 5976-80.

46. Lavoie B, Parent A. Pedunculopontine nucleus in the squirrel monkey: cholinergic and glutamatergic projections to the substantia nigra. J Comp Neurol 1994; 344: 232-41.

47. Shink E, Sidibé $M$, Smith Y. Efferent connections of the internal globus pallidus in the squirrel monkey: II. Topography and synaptic organization of pallidal efferents to the pedunculopontine nucleus. J Comp Neurol 1997; 382: 348-63.

48. Tsang EW, Hamani C, Moro E, et al. Involvement of the human pedunculopontine nucleus region in voluntary movements. Neurology 2010; 75: 950-9.

49. Litvan I, Blesa R, Clark K, et al. Pharmacological evaluation of the cholinergic system in progressive supranuclear palsy. Ann Neurol 1994; 36: 55-61.

50. Nebes RD, Pollock BG, Halligan EM, Kirshner MA, Houck PR. Serum anticholinergic activity and motor performance in elderly persons. J Gerontol A Biol Sci Med Sci 2007; 62: 83-5.

51. Zesiewicz TA, Sullivan KL. Treatment of ataxia and imbalance with varenicline (Chantix): report of 2 patients with spinocerebellar ataxia (types 3 and 14). Clin Neuropharmacol 2008; 31: 363-5.

52. Alves G, Larsen JP, Emre M, Wentzel-Larsen T, Aarsland D. Changes in motor subtype and risk for incident dementia in Parkinson's disease. Mov Disord 2006; 21: 1123-30.

53. Taylor JP, Rowan EN, Lett D, O'Brien JT, McKeith IG, Burn DJ. Poor attentional function predicts cognitive decline in patients with non-demented Parkinson's disease independent of motor phenotype. J Neurol Neurosurg Psychiatry 2008; 79: 1318-23.

54. Jenkinson N, Nandi D, Miall RC, Stein JF, Aziz TZ. Pedunculopontine nucleus stimulation improves akinesia in a Parkinsonian monkey. Neuroreport 2004; 15: 2621-4. 
55. Breit S, Lessmann L, Unterbrink D, Popa RC, Gasser T, Schulz JB. Lesion of the pedunculopontine nucleus reverses hyperactivity of the subthalamic nucleus and substantia nigra pars reticulata in a 6-hydroxydopamine rat model. Eur J Neurosci 2006; 24: 2275-82.

56. Rauch F, Schwabe K, Krauss JK. Effect of deep brain stimulation in the pedunculopontine nucleus on motor function in the rat 6-hydroxydopamine Parkinson model. Behav Brain Res 2010; 210: 46-53.

57. Nandi D, Aziz TZ, Giladi N, Winter J, Stein JF. Reversal of akinesia in experimental parkinsonism by GABA antagonist microinjections in the pedunculopontine nucleus. Brain 2002; 125: 2418-30.

58. Mazzone P, Lozano A, Stanzione P, et al. Implantation of human pedunculopontine nucleus: a safe and clinically relevant target in Parkinson's disease. Neuroreport 2005; 16: 1877-81.

59. Plaha P, Gill SS. Bilateral deep brain stimulation of the pedunculopontine nucleus for Parkinson's disease. Neuroreport 2005; 16: 1883-7.

60. Stefani A, Lozano AM, Peppe A, et al. Bilateral deep brain stimulation of the pedunculopontine and subthalamic nuclei in severe Parkinson's disease. Brain 2007; 130: 1596-607.

61. Ferraye MU, Debû B, Fraix V, et al. Effects of pedunculopontine nucleus area stimulation on gait disorders in Parkinson's disease. Brain 2010; 133: 205-14.

62. Stefani A, Galati S, Pierantozzi M, et al. Motor and nonmotor effects on PPN DBS in PD patients: insights form intra-operative electrophysiology. The Basal Ganglia IX, 2009; 58: 573-8.

63. Pierantozzi M, Palmieri MG, Galati S, et al. Pedunculopontine nucleus deep brain stimulation changes spinal cord excitability in Parkinson's disease patients. J Neural Transm 2008; 115: 731-5.

64. Ballanger B, Lozano AM, Moro E, et al. Cerebral blood flow changes induced by pedunculopontine nucleus stimulation in patients with advanced Parkinson's disease: a [(15)O] H2O PET study. Hum Brain Mapp 2009; 30: 3901-9.

65. Uyanikgil Y, Balcioglu HA. Neural stem cell therapy in neurological diseases. Arch Med Sci 2009; 5: 296-302.

66. Fiandaca MS, Bankiewicz KS. Gene therapy for Parkinson's disease: from non-human primates to humans. Curr Opin Mol Ther 2010; 12: 519-29.

67. Freed CR, Greene PE, Breeze RE, et al. Transplantation of embryonic dopamine neurons for severe Parkinson's disease. N Engl J Med 2001; 344: 710-9.

68. Carlsson T, Carta M, Muñoz A, et al. Impact of grafted serotonin and dopamine neurons on development of L-DOPA-induced dyskinesias in parkinsonian rats is determined by the extent of dopamine neuron degeneration. Brain 2009; 132: 319-35.

69. Marks WJ Jr, Bartus RT, Siffert J, et al. Gene delivery of AAV2-neurturin for Parkinson's disease: a double-blind, randomised, controlled trial. Lancet Neurol 2010; 9: 1164-72.

70. Marks WJ Jr, Ostrem JL, Verhagen L, et al. Safety and tolerability of intraputaminal delivery of CERE-120 (adenoassociated virus serotype 2-neurturin) to patients with idiopathic Parkinson's disease: an open-label, phase I trial. Lancet Neurol 2008; 7: 400-8.

71. Cho ZH, Han JY, Hwang SI, et al. Quantitative analysis of the hippocampus using images obtained from 7.0 T MRI. Neuroimage 2010; 49: 2134-40.

72. Bledsoe JM, Kimble CJ, Covey DP, et al. Development of the Wireless Instantaneous Neurotransmitter Concentration System for intraoperative neurochemical monitoring using fast-scan cyclic voltammetry. J Neurosurg 2009; 111: 712-23.

73. Andrews RJ. Neuromodulation: advances in the next five years. Ann N Y Acad Sci 2010; 1199: 204-11.

74. Moro E, Hamani C, Poon YY, et al. Unilateral pedunculopontine stimulation improves falls in Parkinson's disease. Brain 2010; 133: 215-24.

75. Pereira EA, Muthusamy KA, De Pennington N, et al. Deep brain stimulation of the pedunculopontine nucleus in Parkinson's disease. Preliminary experience at Oxford. Br J Neurosurg 2008; 22 Suppl 1: S41-4.

76. Peppe A, Pierantozzi M, Chiavalon C, et al. Deep brain stimulation of the pedunculopontine tegmentum and subthalamic nucleus: effects on gait in Parkinson's disease. Gait Posture 2010; 32: 512-8. 